\title{
Molecular Mechanisms of Ischemic Preconditioning with Cardiovascular Aging in Elderly Patients with Arterial Hypertension
}

\author{
Elena A. Kartashova, $\mathrm{PhD}^{1}$; Irina V. Sarvilina, $\mathrm{PhD}, \mathrm{ScD}^{* 2}$ \\ ${ }^{1}$ Rostov-on-Don State Medical University, Rostov-on-Don, Russian Federation \\ ${ }^{2}$ Medical Centre "Novomeditsina", Rostov-on-Don, Russian Federation
}

\begin{abstract}
The purpose of this study was to analyze molecular mechanisms of ischemic preconditioning (IPC) with cardiovascular aging in elderly patients with isolated systolic hypertension (ISH).

The study included 306 persons divided into two groups: Group 1 (the control group) included 150 elderly patients without $\mathrm{AH}$, and Group 2 (the experimental group) included 156 elderly patients with ISH according to the inclusion/exclusion criteria. The duration of ISH was 13.5 years. All patients received a double combination of antihypertensive drugs. We applied standard methods for identification of ISH and secondary hypertension. Molecular phenotyping of blood plasma with the identification of molecules involved in IPC process were processed with methods of proteomics. The data of the molecular interactions and functional features of proteins were received with STRING 10.0 database. Bioinformatics analysis has revealed the presence of molecules that are the participants in the pathways of IPC, cardiovascular aging and the molecular interactions involved. (Int J Biomed. 2016;6(1):60-64.).
\end{abstract}

Keywords: ischemic preconditioning; cardiovascular aging; isolated systolic hypertension; proteomics; molecular interactions.

\section{Introduction}

Aging is an inevitable part of life and unfortunately poses the largest risk factor for cardiovascular disease. By 2030, approximately $20 \%$ of the population will be aged 65 or older. In this age group, cardiovascular diseases (CVD) will result in $40 \%$ of all deaths and rank as the leading cause [1]. Aging has a remarkable effect on the heart and arterial system, leading to an increase in cardiovascular diseases, including atherosclerosis, hypertension, myocardial infarction (MI), and stroke. Aging cardiovascular tissues are exemplified by pathological alterations, including hypertrophy, altered left ventricular (LV) diastolic function, and diminished LV systolic reverse capacity, increased arterial thickening and stiffness, and impaired endothelial function [2-6]. Isolated systolic hypertension (ISH) is the most common form of hypertension seen in the elderly $[7,8]$ and the most interesting model of aging of the cardiovascular system. Age is a powerful predictor of mortality for patients with acute MI [9-12]. However, until recently, the fields of cardiovascular disease and molecular biology of aging have remained largely separate [1]. The

*Corresponding author: Irina V. Sarvilina, PhD, ScD. CEO of Medical Centre "Novomeditsina», Rostov-on-Don, Russia. E-mail: isarvilina@mail.ru higher mortality and morbidity associated with advancing age could be due to a reduction in some endogenous protective mechanism against myocardial ischemia, a classic example being "ischemic preconditioning" (IPC).

In 1986, Murry and colleagues revealed an intriguing paradox (ie, that one or more brief episodes of ischemia, too brief in themselves to cause myocyte death, render the heart resistant to a later, more prolonged period of coronary artery occlusion) [13]. This concept of "preconditioning with ischemia" has, since its first description, captured the interest of researchers worldwide. Cardiac IPC is represented as an anti-ischemic vaccination. In other words, IPC is a classical example of the hormetic effect of mild stress (i.e. brief and multiple ischemic episodes) which protects the heart against the more prolonged ischemic insult [14-16].

Pre-infarction angina (PIA) is the most evident equivalent of IPC. A retrospective analysis of in-hospital outcomes in the well-known TIMI-4 trial revealed that patients with a history of angina at any time before acute MI had a lower incidence of in-hospital death, congestive heart failure and/or shock, as well as smaller infarct sizes sizes (determined by creatine kinase release) when compared to the cohort without PIA [17]. The age-related reduction of IPC has been successively confirmed in several studies [18-20]. Thus, PIA has been studied in adult and elderly patients in terms 
of in-hospital primary and secondary events: in adult patients ( $<65$ years), both in-hospital mortality and cardiogenic shock were more frequent in the absence than in the presence of PIA; CK-MB (creatine kinase myoglobin fraction) peak, transmural infarctions number, the incidence of ventricular tachycardia and fibrillation, and the ventricular dysfunction were significantly higher in the adult patients without than in those with PIA. In elderly patients ( $\geq 65$ years), the protective effect of PIA angina seems to be lost: both in-hospital primary and secondary end-points were similar in elderly patients with and without PIA. Logistic regression, adjusted for several variables including the use of thrombolytic and anti-anginal therapy, demonstrated that PIA is a protective variable against mortality and cardiogenic shock in adult but not in elderly patients [21].

The molecular mechanism of ischemic preconditioning is very complex. From activation of G-protein-coupled receptors (GPCR) by adenosine, norepinephrine, bradykinin, opioids, ect., phosphoinositide-3-kinase (PI3K)/serine/threonine kinase (Akt) is activated with subsequent downstream activation of nitric oxide synthase (NOS) and nitric oxide (NO) formation, and guanylate cyclase, protein kinase $\mathrm{G}(\mathrm{PKG})$ and protein kinase $\mathrm{C}$ (PKC) activation. Mechanisms of the age-related reduction of IP require a thorough study.

Methods of molecular analysis of large interactomes (blood, urine) and human tissues (myocardium, vascular wall), including methods of genomics, transcriptomics, proteomics, and metabolomics, allow us to explore the age-related IPC mechanisms in elderly patients with ISH, as models of cardiovascular aging.

\section{Material and Methods}

The study was prospective comparative cohort with parallel design. The study conducted in accordance with WMA Declaration of Helsinki (1964-2013) and the permission of the Ethics Committee of the Rostov State Medical University. It included 306 persons divided into two groups: Group 1 (the control group) included 150 elderly patients without $\mathrm{AH}$, and Group 2 (the experimental group) included 156 elderly patients (early old-age pensioners, between 65 and 74 years) with ISH according to the inclusion/exclusion criteria. Patients with ISH corresponded to the criteria for the classification of blood pressure levels (SBP $>140 \mathrm{mmHg}$ and $\mathrm{DBP}<90$ $\mathrm{mmHg})$ and the risk stratification - middle $(\mathrm{n}=87)$ and high $(n=69)$ additional risk proposed by the WHO/ISH (1999), Guidelines for hypertension in Russia (2008), 2013 ESH/ESC Guidelines for the management of arterial hypertension [22]. The duration of ISH was 13.5 years. All patients of Group 2 received one of the combinations of antihypertensive drugs (calcium antagonist amlodipine+diuretic indapamide retard; angiotensin receptor blocker valsartan+diuretic indapamide retard; calcium antagonist amlodipine+angiotensin receptor blocker valsartan). The duration of therapy was 5,2 years.

At the stage of data collection and screening, we applied standard methods for identification of ISH and secondary hypertension: the assessment of the patient's complaints, medical history, physical examination, 24-hour ABPM, ECG
(ATES MEDICA, Italy-Russia), echocardiography (SamsungMedison, South Korea), blood and urine tests, biochemical analysis of blood and urine, blood level of aldosterone and corticosteroids, plasma renin activity, urinary catecholamines and metabolites (ELISA, Siemens 2000, Germany), coagulogram («Instrumentation Laboratory», USA), and MRI of adrenal glands, kidney and brain (Philips Intera 1,5T, Japan). For estimating arterial distensibility, cardio-ankle vascular index (CAVI) and heart-brachial pulse wave velocity (B-PWV) were measured non-invasively using the VaSera VS-1000 vascular screening system (Fukuda Denshi, Tokyo, Japan). CAVI was measured at the following segments: CAVI1 (heart to ankle) and CAVI-2 (heart to iliac artery). All measurements were conducted in a room kept at a constant temperature with the subject resting in a supine position after resting for $5 \mathrm{~min}$.

Molecular phenotyping of biosamples (blood plasma) with the identification of molecules involved in IPC process were processed with methods of proteomics: the prefractionation, the separation of proteins with standard sets (MB-HIC C8 Kit, MB-IMAC Cu, MB-Wax Kit, «Bruker», USA), matrix-assisted laser desorption-ionization time-offlight mass spectrometry (MALDI-TOF-MS/MS, Ultraflex II, «Bruker», USA). The partially identified sequences were then submitted to "BLAST protein-protein" and screened against the Homo sapiens Swissprot database to check if this identification matched the MASCOT-identification (Matrix Science). The data of the molecular interactions and functional features of proteins were received with STRING 10.0 database. The analysis of functional activity of molecules in the mechanism of IPC was performed by the databases InterPro, Entrez, SWISS-PROT, NRDB, PDB, and KEGG.

Statistical analysis of the survey data was performed using the software "Statistica 12.0" (Statsoft, Russia). Baseline characteristics were summarized as frequencies and percentages for categorical variables and as mean \pm SEM for continuous variables. Student's unpaired and paired t-tests were used to compare two groups for data with normal distribution. Group comparisons with respect to categorical variables are performed using chi-square tests with Yates correction or, alternatively, Fisher's exact test when expected cell counts were less than 5 . A probability value of $\mathrm{P}<0.05$ was considered statistically significant.

\section{Results}

Clinical and anamnestic characteristics of ISH patients are presented in Table 1. In Group 2, parameters of coagulogram, blood and urine tests, blood levels of glucose, uric acid, potassium, aldosterone and corticosteroids, plasma renin activity, urinary catecholamines and metabolites were in the range of reference values. MRI signs of leukoaraiosis were detected in all patients with ISH.

At the stage of data collection and screening, we noted a significant increase in indicators of 24-h daytime and nighttime SBP and DBP and heart rate in Group 2 patients compared to Group 1 patients (daytime SBP: Group 1-117.6 $\pm 3.5 \mathrm{mmHg}$, and Group 2 - 141.2 $\pm 3.4 \mathrm{mmHg}, P<0.001$; daytime DBP: 
Group $1-69.4 \pm 2.4 \mathrm{mmHg}$ and Group $2-78.4 \pm 1.6 \mathrm{mmHg}$, $P<0.001$; nighttime SBP: Group $1-117.6 \pm 2.9 \mathrm{mmHg}$ and Group $2-130.9 \pm 3.6 \mathrm{mmHg}, P<0.001$; nighttime DBP: Group $1-67.6 \pm 1.8 \mathrm{mmHg}$ and Group $2-81.8 \pm 1.4 \mathrm{mmHg}, \mathrm{P}<0,001$; heart rate: Group 1- 72.3 $\pm 1.2 \mathrm{bpm}$ and Group $2-84.5 \pm 1.5$ bpm, $P<0.001)$ despite the use of antihypertensive drugs.

\section{Table 1.}

Clinical-anamnestic characteristics of the studied patients

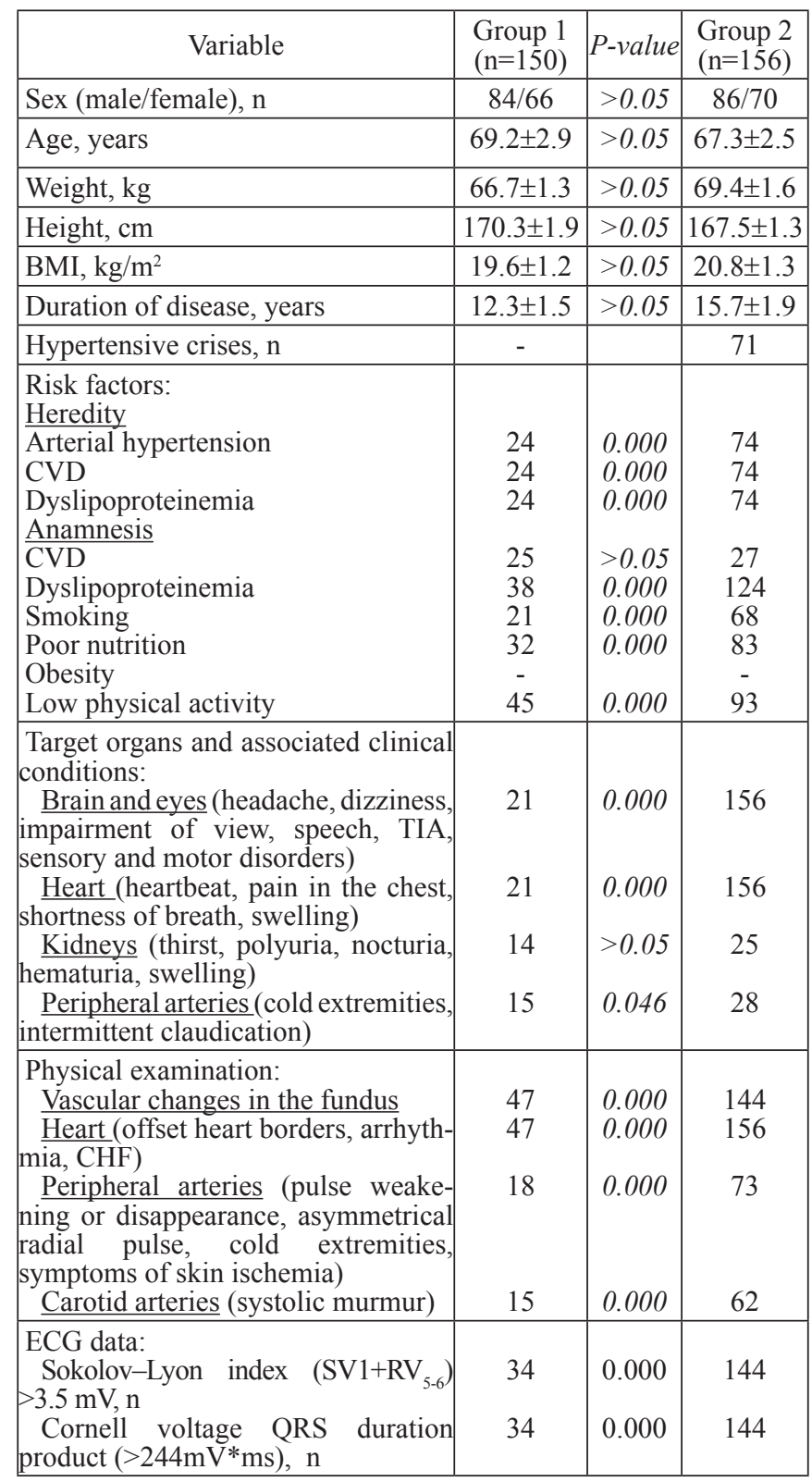

TIA - transient ischemic attack; CHF - chronic heart failure.

The concentric LVH was identified in all ISH patients compared to the control group (LVMI: Group $1-117.4 \pm 3.2 \mathrm{~g}$ / $\mathrm{m}^{2}$ and Group $2-134.3 \pm 4.3 \mathrm{~g} / \mathrm{m}^{2}, P<0.001$; RWT: Group 1$0.45 \pm 0.03$ and Group $2-0.33 \pm 0.01, P<0.001)$.

We also noted significant differences in indicators of arterial stiffness between Groups 1 and 2 (B-PWV: Group $1-6.6 \pm 1.2 \mathrm{~m} / \mathrm{sec}$ and Group $2-10.3 \pm 1.6 \mathrm{~m} / \mathrm{sec}, P<0.01$;
CAVI1: Group 1- 8.0 \pm 0.7 and Group $2-11.2 \pm 1.5, P<0.01$; CAVI2: Group 1- 6.7 \pm 0.7 and Group 2- 10.6 $\pm 1.8, P<0.01)$.. Significant changes in lipid profile were identified in Group 2 patients compared to Group 1 patients (total cholesterol: Group $1-3.86 \pm 0.45 \mathrm{mmol} / 1$ and Group $2-6.43 \pm 0.51 \mathrm{mmol} / 1$, $P<0.05$; LDL cholesterol: Group 1- $2.18 \pm 0.39 \mathrm{mmol} / \mathrm{l}$ and Group $2-3.89 \pm 0.31 \mathrm{mmol} / 1, \mathrm{P}<0.05)$.

Proteomic analysis helps in the detection of differences in the component composition of blood plasma proteins involved in IPC in ISH patients compared with the control group (Table 2). Bioinformatics analysis has revealed the presence of molecules that are the participants in the pathways of IPC, cardiovascular aging and the molecular interactions involved.

\section{Discussion}

Proteomic analysis has revealed an increase in the absolute number of ISH patients with an abnormal profile of blood proteins performing certain biological functions and having various localizations in the intra- and extracellular spaces (Table 2). Molecules interact among themselves and with other molecules as participants of age-related IPC in ISH patients.

A significant reduction in the expression of dishevelledassociated activator of morphogenesis 1 (Daam1) in blood plasma indicates the remodeling process in the cardiovascular system through the pathological WNT-pathway. This protein promotes the incorporation of profilin in the cell membrane of cardiomyocytes, which that increase the rigidity and decrease the elasticity of the cardiovascular wall.

APOD is known to regulate smooth muscle cells and is found in abundance within atherosclerotic lesions. Paracrine secretion by endothelial cells causes partial downregulation of APOD expression. Additionally, cell contact-dependent Notch signaling plays a role. NOTCH3 contributes to the downregulation of APOD and by itself is sufficient to attenuate APOD transcript expression. Our data indicates the special role of APOD in the development of age-related IPC [23].

Myocardial ischemic preconditioning upregulated protein 1 (Mipu1) is a newly discovered upregulated gene produced in rats during the myocardial ischemic preconditioning process. Mipul is a nuclear factor with a variety of biological functions, such as participation in the process of myocardial ischemic preconditioning, protection of the myocardium from ischemic disease, and inflammation [24]. Analysis of the function of Mipu1, as well as vascular endothelial growth factor-A (an important neuroprotectant) and ACE (a central component of the renin-angiotensin system) in CVD is beneficial because it may provide new ideas for prevention of cardiovascular aging.

Each protein molecule in the functional group interacts with other protein molecules. For example, the molecular interactions of peroxisome proliferator-activated receptors D (PPARD) are presented in Fig. 1. The concentration of PPARD decreases in the blood plasma of ISH patients compared to the control group. This fact with increasing age means there is a violation of the process of $\beta$-oxidation of fatty acids in 
cardiomyocytes, and a lack of myocyte protection from apoptosis induced by oxidative stress. The decrease in the PPARD expression in the blood plasma of ISH patients can lead to the development of myocardial damage. In our study we found a significant decrease in the expression of endothelial nitric oxide synthase 3 (NOS3) in the blood plasma of ISH patients, leading to a reduction in compensatory vasodilation and angiogenesis, that is associated with cardiovascular aging and features of age-related IPC. We also registered a high expression of endothelin-1 in the blood plasma of ISH patients, which plays a key role in the holding of vascular endothelium homeostasis. This protein reveals a vasoconstrictive effect, induces the accumulation of collagen and stimulates the mitogenesis of fibroblasts (ie, cellular processes underlying in cardiovascular aging).

\section{Conclusion}

The dynamics in the proteome-map of blood serum in ISH patients revealed the molecular features of age-related IPC as the component of the universal pathway of cardiovascular aging.

Table 2.

Qualitative profile of blood plasmain the studied patients

\begin{tabular}{|l|c|c|c|c|l|}
\hline \multicolumn{1}{|c|}{ Protein name } & $\begin{array}{l}\text { Group 1 } \\
(\mathrm{n}=150)\end{array}$ & $\begin{array}{c}\text { Goup 2 } \\
\mathrm{n}=156)\end{array}$ & P-value & $\begin{array}{c}\text { Molecular } \\
\text { weight } \\
(\mathrm{Da})\end{array}$ & (sources: InterPro, Entrez, SWISS-PROT, NRDB, PDB, KEGG) \\
\hline $\begin{array}{l}\text { Disheveled-associated activator of } \\
\text { morphogenesis 1 }\end{array}$ & 83 & 41 & 0.000 & 123396 & Epidermal cell proliferation, and glucose and lipid metabolism \\
\hline $\begin{array}{l}\text { Apolipoprotein D } \\
\begin{array}{l}\text { Myocardial ischemic precon- } \\
\text { ditioning upregulated protein 1 }\end{array}\end{array}$ & 37 & 42 & 0.7518 & 21262 & Fatty acid and steroid metabolism \\
\hline Gamma butyrobetaine hydroxylase & 105 & 82 & 0.0018 & 44687 & $\begin{array}{l}\text { L-carnitine biosynthesis pathway, } \\
\text { mitochondrial beta oxidation }\end{array}$ \\
\hline Endothelial growth factor A & 142 & 125 & 0.0001 & 27042 & Vasculogenesis, neovascular age-related macular degeneration \\
\hline Angiotensin-converting enzyme & 54 & 68 & 0.1753 & 149715 & The convertion of vasoactive peptides \\
\hline Hypoxia inducible factor 1 & 54 & 23 & 0.0000 & 92670 & Transcription factor, regulator the hypoxia in cells \\
\hline $\begin{array}{l}\text { Peroxisome proliferator-activated } \\
\text { receptors D }\end{array}$ & 65 & 38 & 0.0004 & 49903 & $\begin{array}{l}\text { Nuclear hormone receptor, integrator of transcription repression and } \\
\text { nuclear receptor signaling }\end{array}$ \\
\hline Nitric oxide synthase 3, endothelial & 69 & 32 & 0.0000 & 133289 & $\begin{array}{l}\text { Vascular tone, cellular proliferation, leukocyte adhesion, platelet } \\
\text { aggregation }\end{array}$ \\
\hline Endothelin I & 44 & 96 & 0.0000 & 24425 & Vasoconstrictor, vascular homeostasis \\
\hline
\end{tabular}

\section{Competing interests}

The authors declare that they have no competing interests.

\section{References}

1. North BJ, Sinclair DA. The intersection between aging and cardiovascular disease. Circ Res. 2012;110(8):1097-108.

2. Lakatta EG, Levy D. Arterial and cardiac aging: major shareholders in cardiovascular disease enterprises, part I: aging arteries: a "set up" for vascular disease. Circulation. 2003; 107: 139-146.

3. Lakatta EG, Levy D. Arterial and cardiac aging: major

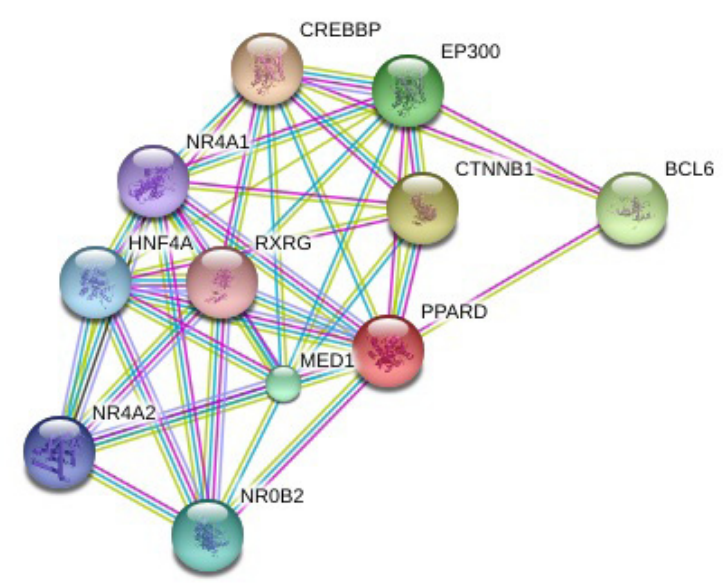

Fig. 1. Molecular interactions of PPARD (STRING 10.0 database)

PPARD, peroxisome proliferator-activated receptor delta; CREBBP, CREB binding protein; CTNNB1, catenin (cadherinassociated protein), beta 1; BCL 6, B-cell CLL/lymphoma 6; EP300, E1A binding protein p300; MED1, mediator complex subunit 1; NROB2, nuclear receptor subfamily 0 , group $B$, member 2; HNF4A, hepatocyte nuclear factor 4, alpha; NR4A2, nuclear receptor subfamily 4, group A, member 2; NR4A1, nuclear receptor subfamily

4, group A, member 1; $\boldsymbol{R} \boldsymbol{X} \boldsymbol{R} \boldsymbol{G}$, retinoid X receptor, gamma. 
Cockcroft, J. Normal vascular aging: differential effects on wave reflection and aortic pulse wave velocity: the AngloCardiff Collaborative Trial (ACCT) / C. McEniery, I. Hall, A. Qasem, I. Wilkinson, J. Cockcroft // J. Am. Coll. Cardiol. 2005. - Vol.46. - № 9. - P. 1753-1760.

7. Basile, J. Hypertension in the elderly: a review of the importance of systolic blood pressure elevation / J. Basile // J. Clin. Hypertens. (Greenwich). 2002. Vol. 4. № 2. P. 108-112. 8. Kearney, P., Whelton, M., Reynolds, K., Muntner, P., Whelton, P., He, J. Global burden of hypertension: analysis of worldwide data / P. Kearney, M.Whelton, K.Reynolds, P.Muntner, P. Whelton, J. He // Lancet. 2005. Vol. 365. № 9455. P. 217-223.

9. Abete P, Testa G, Cacciatore F, Della-Morte D, Galizia G, Langellotto A,et al. Ischemic preconditioning in the younger and aged heart. Aging Dis. 2011 Apr;2(2):138-48.

10. Tresch DD, Brady WJ, Aufderheide TP, Lawrence SW, Williams KJ. Comparison of elderly and younger patients with out-of-hospital chest pain. Arch Intern Med. 1996;156:1089-93.

11. Berger AK, Radford MJ, Wang Y, Krumholz HM. Thrombolytic therapy in older patients. J Am Coll Cardiol. 2000;36:366-74.

12. Boersma E, Pieper KS, Steyerberg EW, Wilcox RG, Chang WC, Lee KL, et al. Predictors of outcome in patients with acute coronary syndromes without persistent ST-segment elevation. Results from an international trial of 9461 patients The PURSUIT Investigators. Circulation. 2000;101:2557-67. 13. Murry CE, Jennings RB, Reimer KA. Preconditioning with ischemia: a delay of lethal cell injury in ischemic myocardium. Circulation 1986;74:1124-1136.

14. Kloner RA, Bolli R, Marban E, Reinlib L, Braunwald E. Medical and cellular implications of stunning, hibernation and preconditioning. An NHLBI workshop. Circulation. 1998;97:1848-67.

15. Napoli C, Pinto A, Cirino G. Pharmacological modulation, preclinical studies, and new clinical features of myocardial ischemic preconditioning. Pharmacol Ther. 2000;88:311-31.

16. Yellon DM, Downey JM. Preconditioning the myocardium: from cellular physiology to clinical cardiology. Physiol Rev. 2003;83:1113-51.

17. Kloner RA, Shook T, Przyklenk K et al. Previous angina alters in-hospital outcome in TIMI-4: a clinical correlate to preconditioning? Circulation 1995;91:37-47.

18. Fenton, R., Dickson, E., Meyer, T,. Dobson JG., Jr. Aging reduces the cardioprotective effect of ischemic preconditioning in rat heart J. Mol. Cell. Cardiol. - 2000. - №32. - P.13711375.

19. Bartling B, Friedrich I, Silber RE, Simm A. Ischemic preconditioning is not cardioprotective in senescent human myocardium. Ann Thorac Surg.2003;76:105-11. [PubMed]

20. Boengler K, Konietzka I, Buechert A, Heinen Y, Garcia-Dorado D, Heusch G, Schulz R, et al. Loss of ischemic preconditioning's cardioprotection in aged mouse hearts is associated with reduced gap junctional and mitochondrial levels of connexin 43. Am J Physiol Heart Circ Physiol. 2007;292:H1764-9.

21. Abete P, Ferrara N, Cacciatore F, Sagnelli E, Manzi $\mathrm{M}$, Carnovale V, et al. Angina-induced protection against myocardial infarction in adult and senescent patients. A loss of preconditioning mechanism in aging heart. J Am Coll Cardiol. 1997;30:947-54.

22. Mancia G, Fagard R, Narkiewicz K, Redon J, Zanchetti A, Böhm M, et al. $2013 \mathrm{ESH} / \mathrm{ESC}$ guidelines for the management of arterial hypertension: the Task Force for the Management of Arterial Hypertension of the European Society of Hypertension (ESH) and of the European Society of Cardiology (ESC). Eur Heart J. 2013;34(28):2159-219.

23. Pajaniappan M, Glober NK, Kennard S, Liu H, Zhao N, Lilly B.Endothelial cells downregulate apolipoprotein $\mathrm{D}$ expression in mural cells through paracrine secretion and Notch signaling. Am J Physiol Heart Circ Physiol. 2011; 301(3): H784-H793.

24. Han D, Zhang C, Fan WJ, Pan WJ, Feng DM, Qu SL, et al. Myocardial ischemic preconditioning upregulated protein 1(Mipu1):zinc finger protein 667 - a multifunctional KRAB/ C2H2 zinc finger protein. Braz J Med Biol Res. 2015; 48(1): 1-5. 